\title{
The Prevalence of the Potential Drug-Drug Interactions Involving Anticancer Drugs in China: A Retrospective Study
}

\author{
Weilan WANG ${ }^{1}$, Bingkun XIAO ${ }^{2}$, Ziqi LIU ${ }^{3}$, Dongxiao WANG ${ }^{1},{\text { Man } Z H U^{1}}^{1}$ \\ 1. Department of Pharmacy, General Hospital of People's Liberation Army (PLA), Beijing, 100853, China \\ 2. Department of Pharmacochemistry, Institute of Radiation Medicine, Beijing, 100850, China \\ 3. Department of Pharmacy, General Hospital of People's Liberation Army (PLA) Rocket Force, Beijing, 100088, China \\ *Corresponding Author: Email: 13661282643@163.com
}

(Received 11 Nov 2017; accepted 26 Mar 2018)

\begin{abstract}
Background: To survey the prevalence of potential drug-drug interactions (DDIs) between anticancer drugs and non-anticancer drugs and evaluate the risk factors associated with these drug-drug interactions in China. Methods: All discharged patients in the Department of Oncology were collected from Jun to Dec in 2016 with the Hospital Information System of the Chinese people's Liberation Army General Hospital. Drugs were screened for interactions by Micromedex solutions database. Descriptive statistics were generated and logistic regression was used to identify the associated factors.

Results: Among 6578 eligible patients, 1979 potential drug interactions were found in 1830 patients (27.82\%). The most common drug-drug interaction was cisplatin and furosemide. Erlotinib was most likely to interact with various non-anticancer drugs. Most interactions were classified as pharmacodynamics $(71.60 \%)$, major severity $(97.02 \%)$ and were supported by fair documentation evidence $(86.21 \%)$. In multivariate analysis, increasing number of medications, lung cancer and patients with stage IV had a higher risk for potential drugdrug interactions.

Conclusion: Potential drug-drug interactions between antineoplastic drugs and non-antineoplastic drugs occur frequently in cancer patients of Chinese hospitals. Doctors should fully consider potential risk associated with DDIs. Further research should be performed to evaluate real clinical significance of these drug-drug interactions.
\end{abstract}

Keywords: Anticancer drugs; Drug-drug interactions; Prevalence; China

\section{Introduction}

Drug-drug interactions (DDIs) are defined as a medication interferes with pharmacokinetic, pharmacodynamic, or pharmaceutical properties of another drug, resulting in an altered net effect of one or both drugs (1). DDIs could be a significant cause of morbidity and mortality because they may result in enhancement of drug toxicity and decrease in therapeutic effects of drugs (2-4). DDIs are of particular concern in oncology be- cause anticancer drugs usually have a narrow therapeutic index and small changes in cytotoxic activity due to a DDI can have serious consequences (5). For example, concurrent use of nonsteroidal anti-inflammatory drugs (NSAIDs) and methotrexate may result in an increased risk of methotrexate toxicity, and fatal cases have been reported $(6,7)$. Dual CYP3A4 and CYP1A2 inhibitor ciprofloxacin may result in increased erlo- 
tinib exposure and even lead to death (8). Patients receiving anti-cancer therapy are particularly vulnerable to DDIs because they often take numerous medications concurrently to manage their malignancy, toxicities, cancer-associated syndromes and other co-morbid illnesses. These multiple medications can increase the risk of DDIs markedly $(9,10)$.

However, very limited data are available for potential drug interactions associated with anticancer therapy. In Belgium, forty-one potential interactions involving an anticancer agent and considered to be clinically significant were identified among $25 \%$ of patients (11). Overall, 5\% of patients taking oral anticancer agents in Singapore were exposed to $\geq 1$ potentially interacting drug combination (10). An observational study using medical records and autopsy analysis showed that $4 \%$ of deaths among cancer patients were caused by chemotherapy itself, and serious drug-drug interactions were sometimes suspected (12).

To date, there was no relevant research data about DDIs involving anticancer agents in China. The Chinese people's liberation army general hospital is the largest comprehensive hospital in China, owning more than 4400 beds in all and more than 300 beds in oncology. Considering combination between antineoplastic drugs and antineoplastic drugs sometimes are unavoidable as standard treatment such as concurrent use of cisplatin and paclitaxel despite enhancement of drug toxicity for DDIs, the main purpose of this study was to survey the prevalence of potential DDIs between antineoplastic drugs and nonantineoplastic drugs and evaluate the risk factors associated with these DDIs in the largest comprehensive hospital in China.

\section{Materials and Methods}

\section{Study subjects}

All discharged patients in the Department of Oncology were collected from Jun to Dec in 2016 with the Hospital Information System of the Chinese people's Liberation Army General Hospital.
The study protocol was approved by the Medical Ethics Committee of the hospital.

\section{Experimental protocol}

The inclusion criteria were: patients who received at least one anti-cancer drug and one nonanticancer drug simultaneously during oncology department stay were considered eligible. Anticancer agents include all traditional anti-tumor agents and molecule-targeted agents, and administration route was defined as intravenous and oral administration route.

For each patient included, we collected the following data from the electronic medical record: age, sex, medications administered concurrently for anti-cancer drugs and non-anti-cancer drugs, type of cancer, tumor stage, and number of medication orders. Micromedex solutions database was utilized to identify potential interactions administered simultaneously between anti-cancer drugs and non-anti-cancer drugs because it was recognized as one of the most useful resources for identifying potential clinical effects (13). Micromedex classifies DDIs into 5 categories of severity: contraindicated, major, moderate, minor, and unknown. For this study, only contraindicated, major and moderate were selected because interactions of minor severity lack clinical significance. Definitions for severity and documentation of DDIs by Micromedex are shown in Table 1. Regarding mechanism of action, DDIs were classified as either pharmacokinetics or pharmacodynamics. Pharmaceutical interactions were not investigated because they were beyond the scope of our study. Tumor stages are classified in the study as stage IV and prior to stage IV because patients with stage IV face more complex physical, psychological, social, and spiritual consequences of disease and more treatment including disease-directed therapy, symptom management, and attention to quality of life compared with prior to stage IV (14-17).

\section{Statistical analysis}

We used descriptive statistics to summarize patient and potential drug interactions characteristics. Continuous variables were reported as 
mean \pm standard deviation (SD). The data were analyzed using independent sample t-test and chisquare test to compare the characteristic differences including age, sex, type of cancer, tumor stage and number of medication orders between patients with and without DDIs. Multiple logistic regression analysis was used to identify the factors from the statistically significant single factors. Data were analyzed using statistical software SPSS version 13.0 (Chicago, IL, USA) with test level $\alpha=0.05$. $P$-values less than 0.05 were considered statistically significant.

\section{Results}

From Jun to Dec in 2016, 6578 patients who received at least one anti-cancer drug and one nonanti-cancer drug simultaneously were identified. The characteristics of the 6578 eligible patients were summarized in Table 2.

Table 1: Micromedex classification criteria for DDIs

\begin{tabular}{|c|c|}
\hline Classification & Definition \\
\hline \multicolumn{2}{|l|}{ Severity } \\
\hline Contraindicated & The drugs are contraindicated for concurrent use \\
\hline Major & $\begin{array}{l}\text { The interaction may be life-threatening and/or require medical intervention to mini- } \\
\text { mize or prevent serious adverse effects }\end{array}$ \\
\hline Moderate & $\begin{array}{l}\text { The interaction may result in exacerbation of the patient's condition and/or require an } \\
\text { alteration in therapy }\end{array}$ \\
\hline Minor & The interaction would have limited clinical effects \\
\hline \multicolumn{2}{|l|}{ Documentation } \\
\hline Excellent & Controlled studies have clearly established the existence of the interaction \\
\hline Good & $\begin{array}{l}\text { Documentation strongly suggests the interaction exists, but well-controlled studies are } \\
\text { lacking }\end{array}$ \\
\hline Fair & $\begin{array}{l}\text { Available documentation is poor, but pharmacologic considerations lead clinicians to } \\
\text { suspect the interaction exists; or, documentation is good for a pharmacologically simi- } \\
\text { lar drug. }\end{array}$ \\
\hline Unknown & Unknown \\
\hline
\end{tabular}

Table 2: Patient characteristics $(\mathrm{n}=6578)$

\begin{tabular}{|c|c|c|}
\hline Characteristics & $\mathbf{N}$ & $\%$ \\
\hline \multicolumn{3}{|l|}{ Sex } \\
\hline Male & 3134 & 47.64 \\
\hline Female & 3444 & 52.36 \\
\hline \multicolumn{3}{|l|}{ Age } \\
\hline Mean \pm SD & $54.82 \pm 11.16$ & \\
\hline Range & $12-86$ & \\
\hline \multicolumn{3}{|c|}{ Number of medication orders } \\
\hline Mean \pm SD & $13.38 \pm 6.83$ & \\
\hline Range & $2-49$ & \\
\hline \multicolumn{3}{|l|}{ Tumor stage } \\
\hline Earlier than IV & 2431 & 36.96 \\
\hline IV & 4147 & 63.04 \\
\hline \multicolumn{3}{|l|}{ Cancer type } \\
\hline Lung & 1664 & 25.30 \\
\hline Breast & 1408 & 21.40 \\
\hline Gastric & 900 & 13.68 \\
\hline Intestinal & 1273 & 19.35 \\
\hline Others * & 1333 & 20.26 \\
\hline
\end{tabular}

* Others include pancreatic cancer, esophageal cancer, liver cancer, ovarian cancer, head and neck cancer, mesothelioma, melanoma, testicular cancer, neuroendocrine tumors, cholangiocarcinoma, adrenal cortical carcinoma, fibrous histiocytoma, prostate cancer, cervical cancer, trophoblastic tumor 
The mean \pm SD age was $55.10 \pm 11.16 \mathrm{yr}$ (range,1286). More than half of the patients $(52.36 \%)$ were females. Most patients (63.04\%) were diagnosed with stage IV tumor. Lung cancer $(25.30 \%)$, breast cancer $(21.40 \%)$, intestinal cancer $(19.35 \%)$ and gastric cancer $(13.68 \%)$ were the most common cancer type. And the mean \pm SD number of medication orders per patient was $13.38 \pm 6.83$ (range, 2-49).

Among 6578 eligible patients, 1979 potential drug interactions were found in 1830 patients $(27.82 \%)$. One DDI was found in 1681 patients $(91.86 \%), 2$ in 149 patients $(8.14 \%)$.
1867(94.34\%) DDIs were identified in traditional anti-tumor agents and $112(5.66 \%)$ DDIs were in molecule- targeted drugs. The severity of majority of DDIs was classified as major $(97.02 \%)$ and only $2.98 \%$ as moderate. DDIs involving contraindication were not found in the study. Documentation evidence of DDIs was classified as fair $(86.21 \%)$, good $(13.34 \%)$ and excellent $(0.45 \%)$. Totally, $562(28.40 \%)$ DDIs were pharmacokinetic and 1417(71.60\%) DDIs were pharmacodynamic in pharmacological mechanism (Table 3). The most common drug-drug interactions are summarized in Table 4.

Table 3: Characteristics of potential drug interactions

\begin{tabular}{lcc}
\hline Characteristics & $\boldsymbol{N}$ & $\boldsymbol{\%}$ \\
\hline Number of enrolled patients & 6578 & \\
Number of patients with $\geq 1$ potential drug interaction & 1830 & 27.82 \\
Number of DDIs & 1979 & \\
Drug subclass of DDIs & 1867 & 94.34 \\
$\quad$ Traditional anti-tumor drugs & 112 & 5.66 \\
$\quad$ Molecule-targeted drugs & & \\
Severity of DDIs & 1920 & 97.02 \\
$\quad$ Major & 59 & 2.98 \\
$\quad$ Moderate & 9 & 0.45 \\
Documentation level of DDIs & 264 & 13.34 \\
$\quad$ Excellent & 1706 & 86.21 \\
$\quad$ Good & & \\
$\quad$ Fair & 562 & 28.40 \\
Pharmacological mechanism of DDIs & 1417 & 71.60 \\
$\quad$ Pharmacokinetic & & \\
$\quad$ Pharmacodynamic &
\end{tabular}

Overall, 1379 out of1979 (69.68\%) DDI was cisplatin and furosemide, accounting for the largest percentage of DDIs. Moreover, erlotinib was most likely to interact with various nonanticancer drugs including PPI, rifampicin, warfarin, and carbamazepine.

Table 5 shows the characteristics of patients with and without DDIs. Patients with DDIs were more likely to be male, increasing number of medications, cancer type, and patients with stage IV (all, $P<0.001)$. No significant differences were found in age $(P>0.05)$. In multivariable analysis, increasing number of medications (odds ratio $[\mathrm{OR}]=1.09,95 \%$ CI 1.08-1.11), cancer type (OR for lung vs. gastric tumors $=4.59,95 \%$ CI 3.79 5.56 , other types of cancer vs. gastric tumors $=2.03,95 \% \mathrm{CI} 1.66-2.48)$ and patients with stage IV $(\mathrm{OR}=1.52,95 \% \mathrm{CI} 1.32-1.76)$ remained significant associated with potential drug interactions. However, no significant differences were found in sex. Results of the multiple logistic regression analysis are presented in Table 6. 
Table 4: The most common drug-drug interactions in this study population ( $\mathrm{n}=1979)$

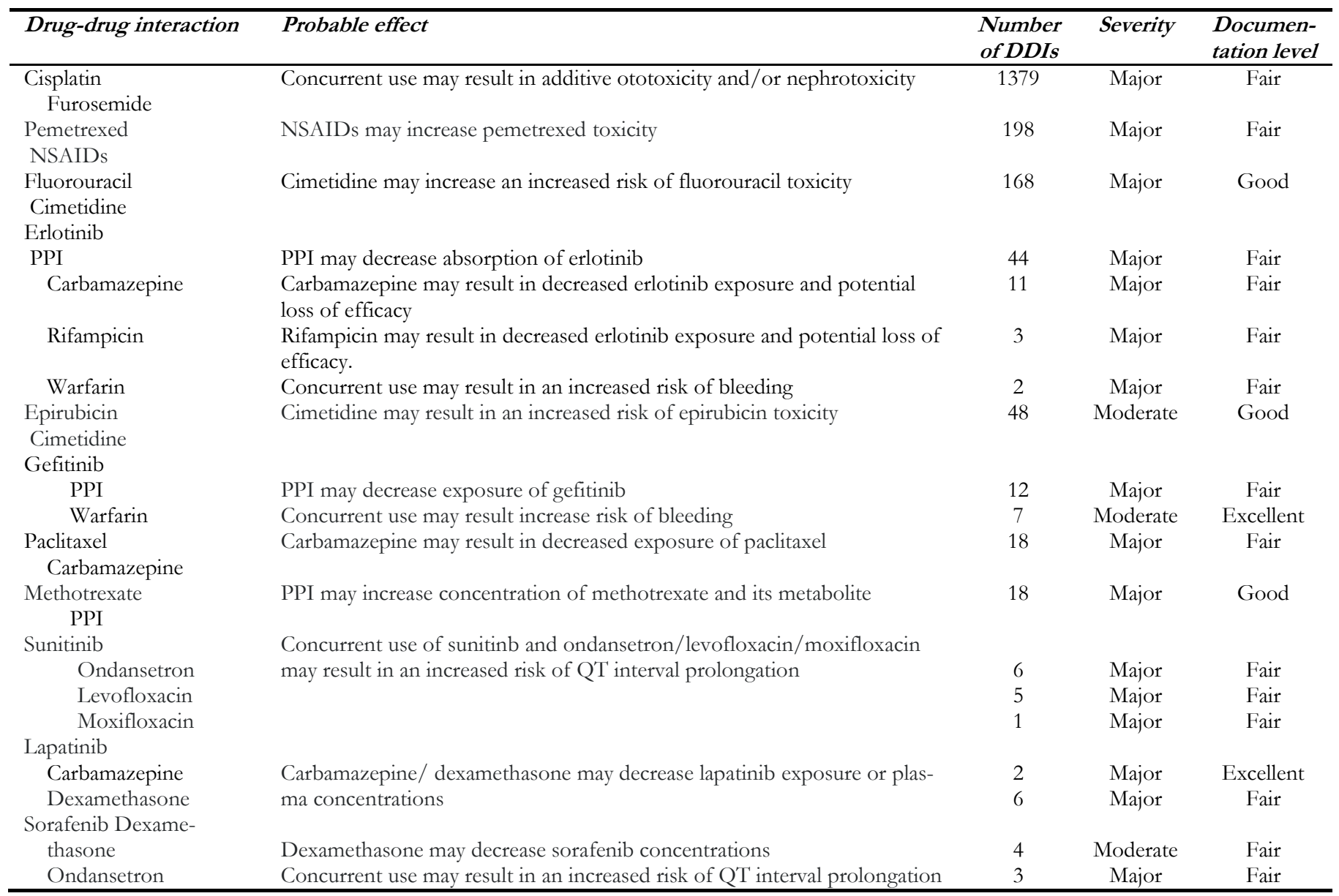

Table 5: Characteristics of patients with and without DDIs $(\mathrm{n}=6578)$

\begin{tabular}{|c|c|c|c|}
\hline Characteristics & $\begin{array}{c}\text { Patients with DDIs } \\
(n=1830)\end{array}$ & $\begin{array}{c}\text { Patients without DDIs } \\
(n=4748)\end{array}$ & $P$ \\
\hline Sex & & & $<0.001$ \\
\hline Male, n (\%) & $1153(63.01 \%)$ & $1981(41.72 \%)$ & \\
\hline Female, n (\%) & $677(36.99 \%)$ & $2767(58.28 \%)$ & \\
\hline Age & & & $>0.05$ \\
\hline Mean \pm SD & $55.48 \pm 11.81$ & $54.95 \pm 10.89$ & \\
\hline Range & $12-86$ & $13-85$ & \\
\hline Number of medications & $<0.001$ & & \\
\hline Mean \pm SD & $16 \pm 6.39$ & $12 \pm 6.51$ & \\
\hline Range & 3-58 & $2-49$ & \\
\hline Cancer type & & & $<0.001$ \\
\hline Lung & $935(51.09 \%)$ & $729(15.35 \%)$ & \\
\hline Breast & $134(7.32 \%)$ & $1274(26.83 \%)$ & \\
\hline Gastric & $204(11.15 \%)$ & $696(14.66 \%)$ & \\
\hline Intestinal & $18(0.98 \%)$ & $1255(26.43 \%)$ & \\
\hline Others * & $794(16.72 \%)$ & $539(29.45 \%)$ & \\
\hline Tumor stage & & & $<0.001$ \\
\hline Earlier than IV & $476(26.01 \%)$ & $1955(41.18 \%)$ & \\
\hline IV & $1354(73.99 \%)$ & $2793(58.82 \%)$ & \\
\hline
\end{tabular}

* Others include pancreatic cancer, esophageal cancer, liver cancer, ovarian cance, head and neck cancer, mesothelioma, melanoma, testicular cancer, neuroendocrine tumors, cholangiocarcinoma, adrenal cortical carcinoma, fibrous histiocytoma, prostate cancer, cervical cancer, trophoblastic tumor 
Table 6: Multivariable analysis for factors associated with DDIs

\begin{tabular}{lcc}
\hline Variable & Odds Ratio(95\% CI) & $\boldsymbol{P}$ \\
\hline Sex & $1.11(0.96-1.27)$ & $>0.05$ \\
Number of medications & $1.09(1.08-1.11)$ & $<0.001$ \\
Cancer type & & \\
$\quad$ Gastric & Referent & $<0.001$ \\
Lung & $4.59(3.79-5.56)$ & \\
Breast & $0.73(0.56-0.96)$ & \\
Intestinal & $0.05(0.03-0.09)$ & \\
$\quad$ Others $*$ & $2.03(1.66-2.48)$ & $<0.001$ \\
Tumor stage & $1.52(1.32-1.76)$ & \\
\hline
\end{tabular}

$*$ Others include pancreatic cancer, esophageal cancer, liver cancer, ovarian cancer, head and neck cancer, mesothelioma, melanoma, testicular cancer, neuroendocrine tumors, cholangiocarcinoma, adrenal cortical carcinoma, fibrous histiocytoma, prostate cancer, cervical cancer, trophoblastic tumor

\section{Discussion}

Overall, 1830 out of $6578(27.82 \%)$ patients receiving antineoplastic therapy are exposed to at least one potential DDI. The prevalence observed in the study was comparable to the study of Belgium, 25\% of patients were exposed to clinically significant DDIs (11). Considering high risk associated with DDIs in cancer patients, and almost all DDIs $(97.02 \%)$ were classified as major, more attention should be paid by oncologists. Among the identified DDIs, the largest number of drug-drug interaction was cisplatin and furosemide. Furosemide is commonly used to mitigate nephrotoxicity of cisplatin. However, the protective effect of furosemide against nephrotoxicity has not been confirmed (18). In fact, furosemide enhanced nephrotoxicity of cisplatin (19-22). Twenty-six mg median dose of furosemide was associated with cisplatin nephrotoxicity and an explanation is that furosemide may have a direct toxic effect on the kidney (19). Furosemide-induced GFR reduction (20). Highdose furosemide leads to proximal tubular necrosis and its use with cisplatin may aggravate the nephrotoxicity $(21,22)$. In addition, furosemide causes edema of the stria vascularis, disrupts the blood-ear barrier, and enhances the entry of ototoxic drugs into the inner ear, known to potentiate cisplatin-induced hearing loss (23-25). Therefore, many researchers have sought less toxic methods for administering cisplatin without furosemide $(26,27)$. The second most frequent DDI detected in our study was pemetrexed and NSAIDs, mainly found in patients with lung adenocarcinoma with bone metastasis or pain. Severe hematologic toxicities in patients receiving carboplatin-based pemetrexed may be significantly induced by the inhibition of renal tubular secretion of pemetrexed through drug-drug interactions between NSAIDs and pemetrexed (28). Renal dysfunction may easily develop as a result of continued pemetrexed administration combined with NSAID therapy (29). Therefore, it is necessary to take precautions against adverse side effects when combining pemetrexed with NSAID therapy. Erlotinib was most likely to interact with various non-anticancer drugs including PPI, rifampicin, warfarin, and carbamazepine. Erlotinib is a tyrosine kinase inhibitor, which drug interactions occur commonly because of DDIs concern absorption (incomplete drug absorption is a risk of drug interaction) and metabolization by the cytochrome P450 isozymes (30). Significant clinical consequences have been associated with these interaction mechanisms (8, $31,32)$. Interaction between sunitinib and ondansetron or quinolones was found in the study which may result in an increased risk of QT interval prolongation. Similar risk of interaction involved sorafenib and ondansetron. Medical oncologists should be better known about the risk of increased tyrosine kinase inhibitor toxicity or decreased tyrosine kinase inhibitor efficacy in patients given tyrosine-kinase inhibitors. If possible, the combination of some tyrosine-kinase in- 
hibitors and proton-pump inhibitor should be avoided. Dose adjustments of tyrosine-kinase inhibitors are highly recommended when combined with strong CYP3A4 inhibitors or inducers which can significantly affect the exposure to tyrosine-kinase inhibitors. Unless absolutely necessary, coadministration QTc- prolonging tyrosinekinase inhibitors and drugs that prolong the QTc interval should be avoided (30).

Our study revealed that patients taking more medications, those with stage IV and lung cancer patients were at greater risk of drug interactions. Patients with lung cancer were almost 5 times more likely to be exposed to drug interactions than patients with gastric cancer. The finding of Holland researchers was similar to ours. Lung cancer patients have a high risk of drug-drug interactions (33). Cisplatin, TKI, and pemetrexed which often used by lung cancer patients have a high number of DDIs. Conversely, compared with gastric cancer patients, breast and intestinal patients were only 0.73 and 0.05 times to be exposed to drug interactions. The result suggested breast and intestinal patients were at lower risk of drug interactions.

Similar to other studies $(9,34)$, the increasing number of medications was associated with more potential drug interactions in our study. Cancer patients with co-morbid illnesses or cancerassociated syndromes usually take more medications with potential drug-drug interactions. Patients with stage IV were also at increased risk of drug interactions because they face more treatment including disease-directed therapy, symptom management, and attention to quality of life compared with prior to stage IV.

However, different from previous studies (9, 10, $35,36)$, older patients were not found to have an increased risk of DDI exposure in our study. Patients receiving chemotherapy usually need good or moderate performance status, no matter the older or the younger patients.

The present study has several limitations. First, analysis data was only from one institution, so the result might be vulnerable to institution bias. Second, many patients were administered oral therapy such as tyrosine kinase inhibitors outside the hospital, which resulted in drug-drug interactions in patients receiving tyrosine kinase inhibitors were underestimated. And the data were not analyzed to compare the characteristic differences in antineoplastic category between patients with and without DDIs due to difficult evaluation, because many patients were administered simultaneously traditional anti-tumor agents and molecule-targeted agents. In addition, documentation evidence of majority of DDIs (86.21\%) was classified as fair, suggesting the interactions need more available documentation and wellcontrolled studies.

\section{Conclusion}

Potential drug-drug interactions between antineoplastic drugs and non-antineoplastic drugs occur frequently in cancer patients of Chinese hospitals. Doctors should fully consider potential risk associated with DDIs. Further research should be performed to evaluate real clinical significance of these DDIs.

\section{Ethical considerations}

Ethical issues (Including plagiarism, informed consent, misconduct, data fabrication and/or falsification, double publication and/or submission, redundancy, etc.) had been completely observed by the authors.

\section{Acknowledgments}

The authors thank Yiming LU from Beijing Institute of Radiation Medicine, for his help in statistical method of this study.

\section{Conflict of Interest}

The authors declared that there is no conflict of interest.

\section{References}

1. Miranda V, Fede A, Nobuo M, et al (2011). Ad- 
verse drug reactions and drug interactions as causes of hospital admission in oncology. $J$ Pain Symptom Manage, 42(3):342-53.

2. Becker ML, Kallewaard M, Caspers PW, et al (2007). Hospitalisations and emergency department visits due to drug drug interactions: a literature review. Pharmacoepidemiol Drug Saf, 16(6):641-51.

3. Janković SM, Pejčić AV, Milosavljević MN, et al (2018). Risk factors for potential drug-drug interactions in intensive care unit patients. $J$ Crit Care, 43:1-6.

4. Moura CS, Acurcio FA, Belo NO (2009). Drugdrug interactions associated with length of stay and cost of hospitalization. J Pharm Pharm Sci, 12(3):266-72.

5. Blower P, de Wit R, Goodin S, et al (2005). Drug-drug interactions in oncology: why are they important and can they be minimized? Crit Rev Oncol Hematol, 55(2): 117-42.

6. Beijnen JH, Schellens JH (2004). Drug interactions in oncology. Lancet Oncol, 5(8):489-96.

7. Shiver MB, Hall LA, Conner KB, et al (2014). Cutaneous erosions: a herald for impending pancytopenia in methotrexate toxicity. Dermatol Online J, 20(7): 13030/qt46k975h8.

8. 8.Gass-Jégu F, Gschwend A, Gairard-Dory AC, et al (2016). Gastrointestinal perforations in patients treated with erlotinib: A report of two cases with fatal outcome and literature review. Lung Cancer, 99:76-8.

9. Riechelmann RP, Zimmermann C, Chin SN, et al (2008). Potential drug interactions in cancer patients receiving supportive care exclusively. $J$ Pain Symptom Manage, 35(5):535-43.

10. Ko Y, Tan SL, Chan A, et al (2012). Prevalence of the Coprescription of Clinically Important Interacting Drug Combinations Involving Oral Anticancer Agents in Singapore: A Retrospective Database Study. Clin Ther, 34(8):1696-704.

11. Mouzon A, Kerger J, D'Hondt L, et al (2013). Potential interactions with anticancer agents: a cross-sectional study. Chemotherapy, 59(2):8592.

12. Buajordet I, Ebbesen J, Erikssen J, et al (2001). Fatal adverse drug events: the paradox of drug treatment. J Intern Med, 250(4):327-41.

13. Patel RI, Beckett RD (2016). Evaluation of resources for analyzing drug interactions. JMed Libr Assoc, 104(4):290-5.
14. Peppercorn JM, Smith TJ, Helft PR, et al (2011). American society of clinical oncology statement: toward individualized care for patients with advanced cancer. J Clin Oncol, 29(6):75560.

15. 15.Tokito T, Murakami H, Mori K, et al (2015). Implementation status and explanatory analysis of early advance care planning for Stage IV non-small cell lung cancer patients. Jpn J Clin Oncol, 45(3):261-6.

16. 16.Malin JL, O'Neill SM, Asch SM, et al (2011). Quality of supportive care for patients with advanced cancer in a VA medical center. $J$ Palliat Med, 14(5):573-7.

17. Temel JS1, Greer JA, Muzikansky A, et al (2010). Early palliative care for patients with metastatic non-small-cell lung cancer. $N$ Engl J Med, 363(8):733-42.

18. Cornelison TL, Reed E (1993). Nephrotoxicity and hydration man cisplatin, carboplatin, and ormaplatin. Gynecol Oncol, 50(2):147-58.

19. Sekine I, Yamada K, Nokihara H, et al (2007). Bodyweight change during the first 5 days of chemotherapy as an indicator of cisplatin renaltoxicity. Cancer Sci, 98(9):1408-12.

20. Nagai N, Ogata H (1996). The renal clearance of unchanged cisplatin during furosemide and mannitol diuresis is dependent on glomerular filtration rate in rats. J Pharm Sci, 85(7):720-4.

21. McMurtry RJ, Mitchell JR (1977). Renal and hepatic necrosis after metabolic activation of 2-substituted furans and thiophenes, including furosemide and cephaloridine. Toxicol Appl Pharmacol, 42(2):285-300.

22. Lehane D, Winston A, Gray R et al (1979). The effect of diuretic pre-treatment on clinical, morphological and ultrastructural cisplatinum induced nephrotoxicity. Int J Radiat Oncol Biol Phys, 5(8):1393-9.

23. Clemens E, de Vries AC, Pluijm SF et al (2016). Determinants of ototoxicity in 451 platinumtreated Dutch survivors of childhood cancer: A DCOG late-effects study. Eur I Cancer, 69:77-85.

24. McAlpine D, Johnstone BM (1990). The ototoxic mechanism of cisplatin. Hear Res, 47(3):191203.

25. Li Y, Ding D, Jiang H, et al (2011). Coadministration of cisplatin and furosemide causes 
rapid and massive loss of cochlear hair cells in mice. Neurotox Res, 20(4):307-19.

26. Muraki K, Koyama R, Honma Y, et al (2012). Hydration with magnesium and mannitol without furosemide prevents the nephrotoxicity induced by cisplatin and pemetrexed in patients with advanced non-small cell lung cancer. J Thorac Dis, 4(6):562-8.

27. Bodnar L, Wcislo G, Gasowska-Bodnar A, et al (2008). Renal protection with magnesium subcarbonate and magnesium sulphate in patients with epithelial ovarian cancer after cisplatin and paclitaxel chemotherapy: a randomised phase II study. Eur J Cancer, 44(17):2608-14.

28. Kawazoe H, Yano A, Ishida Y, et al (2017). Non-steroidal anti-inflammatory drugs induce severe hematologic toxicities in lung cancer patients receiving pemetrexed plus carboplatin: A retrospective cohort study. PLoS One, 12(2): e0171066.

29. Sakata Y, Iwamoto Y, Inata J, et al (2012). Adverse events during pemetrexed administration caused by concomitant nonsteroid antiinflammatory therapy. Gan To Kagaku Ryoho, 39(6):927-32.

30. van Leeuwen RW, van Gelder T, Mathijssen RH, et al (2014). Drug-drug interactions with ty- rosine kinase inhibitors: a clinical perspective. Lancet Oncol, 15(8):e315-26.

31. Chu MP, Ghosh S, Chambers CR, et al (2015). Gastric Acid suppression is associated with decreased erlotinib efficacy in non-small-cell lung cancer. Clin Lung Cancer, 16 (1):33-9.

32. ter Heine R, van den Bosch RT, SchaeferProkop CM, et al (2012). Fatal interstitial lung disease associated with high erlotinib and metabolite levels. A case report and a review of the literature. Lung Cancer, 75(3):391-7.

33. Rompelman FM, Smit AA, Franssen EJ, et al (2017). Drug-drug interactions of cytostatics with regular medicines in lung cancer patients. J Oncol Pharm Pract, 23(7):483-490.

34. Hadjibabaie M, Badri S, Ataei S, et al (2013). Potential drug-drug interactions at a referral hematology-oncology ward in Iran: a crosssectional study. Cancer Chemother Pharmacol, 71(6):1619-27.

35. Cruciol-Souza JM, Thomson JC (2006). Prevalence of potential drug-drug interactions and its associated factors in a Brazilian teaching hospital. J Pharm Pharm Sci, 9(3):427-33.

36. Nobili A, Pasina L, Tettamanti M, et al (2009). Potentially severe drug interactions in elderly outpatients: results of an observational study of an administrative prescription database. $J$ Clin Pharm Ther, 34(4):377-86. 